\title{
Predictive Value of Computed Tomography With Coronal Reconstruction in the Right Hemicolectomy With Complete Mesocolic Excision:A Retrospective Study.
}

Hui Yu ( $\nabla$ h4ride@163.com )

Fujian Provincial Cancer Hospital https://orcid.org/0000-0002-9146-1210

Yong Zhuang

Fujian Provincial Cancer Hospital

Jinliang Jian

Fujian Provincial Cancer Hospital

Chunkang Yang

Fujian Provincial Cancer Hospital

Research

Keywords: Colonic neoplasms, Right Hemicolectomy, Computed tomographic, Vascular anatomy, Central Vascular Ligation, Complete Mesocolic Excision, Surgery

Posted Date: February 2nd, 2021

DOI: https://doi.org/10.21203/rs.3.rs-168827/v1

License: (c) (1) This work is licensed under a Creative Commons Attribution 4.0 International License.

Read Full License 


\section{Abstract}

Background:

Understanding vascular anatomy is critical for performing central vascular ligation (CVL) in the right hemicolectomy with complete mesocolic excision (CME). This study was aimed to investigate the predictive value of multi-slice spiral computed tomography (MSCT) with coronal reconstruction in the right hemicolectomy.

Methods:

Eighty patients who underwent right hemicolectomy in our hospital were included. The intraoperative imaging data and the images of MSCT with coronal reconstruction were analyzed retrospectively. The detection rate of the ileocolic vein (ICV) and ileocolic artery (ICA) location and the accuracy of the preoperative prediction of their anatomical relationship were analyzed. The detection rate and preoperative prediction accuracy of the gastrocolic trunk of Henle (GTH), middle colic artery (MCA), and middle colin vein (MCV) were analyzed. The distance from the ICV root to the GTH root (ICV-GTH distance) was measured and analyzed. The maximum distance from the left edge of the superior mesenteric artery (SMA) to the right edge of the superior mesenteric vein (SMV), named "leSMA-reSMV distance," was measured and analyzed too.

Results:

74 (92.5\%) patients had been located both the ICV and ICA roots and determined the anatomical relationship between them by MSCT, of which 72 were confirmed by intraoperative findings $₫$ and the accuracy was $90 \%$ (72/80). GTH was located by MSCT in 75 (93.7\%) patients, and the accuracy was $91.25 \%$ (73/80). MCA was located by MSCT in 47(58.75\%) patients, and the accuracy was $46.25 \%$ (37/80). MCV was located by MSCT in 51 (63.75\%)patients, and the accuracy was $53.75 \%$ (43/80). The ICV-GTH distance was measured in $73(91.2 \%)$ patients, and the mean distance was $4.28 \pm 2.5 \mathrm{~cm}$. The leSMA-reSMV distance was measured in $76(95 \%)$ patients, and the mean distance was $2.21 \pm 0.6 \mathrm{~cm}$.

Conclusions:

MSCT with coronary reconstruction can locate the roots of ICV, ICA, and GTH, predict the boundary of SMA and SMV, analyze the anatomical relationship between them, and estimate the location and range of central ligation region, which are of some predictive value in central vascular ligation in the right hemicolectomy with CME.

\section{Background}

Since the concept of complete mesocolic excision (CME) was brought into the surgical treatment of colon cancer,patients who underwent these surgical procedures had obtained a lower local 5-year recurrence rates(from 6.5-3.6\%)and better cancer-related 5-year survival rates(from 82.1-89.1\%) ${ }^{1}$. 
It is important to note that CME should be operated under the principle of central vascular ligation (CVL) which means nearly full-length skeletonization of the superior mesenteric vessels in right hemicolectomy ${ }^{1}$, which was considered as an extended dissection according to $S$. Toyota's research ${ }^{2}$ and the guidance of Japanese Society for Cancer of the Colon and Rectum (JSCCR) ${ }^{3}$. Compared to D3 resection, CME with CVL appears to have longer specimens and contains more mesentery and lymphnode 4 .

However, a lack of proper understanding of vascular anatomy and central vascular ligation region may lead to intraoperative injury, bleeding, and inadequate lymph node clearance, which increases the difficulty of performing CME with CVL, especially in laparoscopic right hemicolectomy and patients with high BMI.

Surgeons and radiologists had tried to use multidetector computed tomography angiography (MDCT) or three-dimensional CT (3D-CT) to predict vascular anatomical variation, which has been reported to have high sensitivity, specificity, accuracy, and reliability ${ }^{5,6}$.

However, preoperative MDCT angiography or 3D-CT is not routinely used due to radiation risk ${ }^{7-9}$, technical limitations, and increased costs. More importantly, due to the lack of anatomical information other than blood vessels in angiographic images, these techniques can not provide surgeons with sufficient visual prediction of lymph node dissection region, e.g. the location and size of the central ligation region and its relationship with other anatomical structures.

In this study, we attempted to use multi-slice spiral computed tomography (MSCT)with coronal reconstruction techniques to assess the vascular anatomy and central ligation region that need to be explored during the right hemicolectomy.

\section{Materials And Methods}

Intraoperative imaging data and MSCT raw data of 80 patients who underwent right hemicolectomy in our hospital from December 2015 to January 2020 were collected.

The age of the patients ranged from 15 to 87 years, including 42 males and 38 females, with BMI ranging from 16.0 to 23.7 , including 10 cecum cancers, 46 ascending colon cancers, 14 liver flexure colon cancers, and 10 proximal transverse colon cancers. 
Table 1 Patient and tumor characteristics $(n=80)$

\begin{tabular}{|c|c|}
\hline & $n(\%)$ \\
\hline Age median / range (years) & $59(15-87)$ \\
\hline \multicolumn{2}{|l|}{ SEX } \\
\hline Male & $42(52.5 \%)$ \\
\hline Female & $38(47.5 \%)$ \\
\hline $\mathrm{BMI}($ mean $\pm \mathrm{SD}$, range) & $22.5 \pm 3.1(16.0-29.1)$ \\
\hline \multicolumn{2}{|l|}{ Tumor site } \\
\hline Cecum & $10(12.5 \%)$ \\
\hline Ascending colon & $46(57.5 \%)$ \\
\hline Hepatic flexure colon & $14(17.5 \%)$ \\
\hline Proximal transverse colon & $10(12.5 \%)$ \\
\hline \multicolumn{2}{|l|}{ pT category* } \\
\hline TO & $3(3.75 \%)$ \\
\hline T1 & $4(5 \%)$ \\
\hline T2 & $7(8.75 \%)$ \\
\hline T3 & $40(50 \%)$ \\
\hline $\mathrm{T} 4$ & $26(32.5 \%)$ \\
\hline \multicolumn{2}{|l|}{ pN category* } \\
\hline pN1 & $37(46.25 \%)$ \\
\hline pN2 & $33(41.25 \%)$ \\
\hline pN3 & $10(12.5 \%)$ \\
\hline \multicolumn{2}{|l|}{ pM category* } \\
\hline Mo & $71(88.75 \%)$ \\
\hline M1 & $9(11.25 \%)$ \\
\hline \multicolumn{2}{|l|}{ Stage } \\
\hline Stage 0 & $3(3.75 \%)$ \\
\hline Stage I & $8(10 \%)$ \\
\hline
\end{tabular}

* According to the 8th edition of the AJCC/UICC TNM staging system 


\begin{tabular}{|ll|}
\hline & $\mathbf{n}(\%)$ \\
\hline Stage II & $23(28.75 \%)$ \\
\hline Stage III & $35(43.75 \%)$ \\
\hline Stage IV & $11(13.75 \%)$ \\
\hline Surgical approach & \\
\hline D2 dissection & $11(13.75 \%)$ \\
\hline CME & $65(81.25 \%)$ \\
\hline Extended resection & $11(13.8 \%)$ \\
\hline Multivisceral resection & $5(6.25 \%)$ \\
\hline Palliative operation & $4(5 \%)$ \\
\hline * According to the 8th edition of the AJCC/UICC TNM staging system \\
\hline
\end{tabular}

All of the 80 patients had an abdominal contrast-enhanced triple-phase MSCT scan with $0.6 \mathrm{~mm}$ thickness per slice before the operation. MSCT was performed with a high-speed scanner (Brilliance iCT区 Philips Medical Systems(Cleveland)Inc囚Cleveland囚USA) including pre-contrast, arterial phase, portal venous phase, and delayed phase after the injection of iohexol. The venous phase is 30 seconds after the end of the arterial scan.

The original scan data were extracted and reconstructed under coronal position in the portal vein phase with multiplanar reconstruction (MPR), maximum intensity projection (MIP) techniques. MSCT and the coronal-reconstructed image were used to locate the roots of ileocolic vein (ICV), ileocolic artery (ICA), gastrocolic trunk of Henle (GTH), middle colic artery (MCA), and middle colin vein (MCV), and the edge of

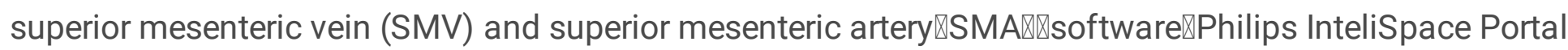
v4.0.4.10004, Phillips Healthcare Nederland B.V.X. The analyzed data were compared with the intraoperative imaging data to evaluate the accuracy. The anatomical relationship between ICV and ICA was analyzed by the coronal-reconstructed image combining with the image of the axial position and MSCT's positioning function. The location of GTH relative to the pancreas and ICV were compared with intraoperative findings to determine their accuracy. The straight line distance from the inferior edge of ICV root to the inferior edge of GTH root (ICV-GTH distance) was measured, and the maximum distance from the left edge of SMA to the right edge of SMV\leSMA-reSMV distance $\$ was measured too. Then the mean distance of the ICV-GTH distance and the leSMA-reSMV distance were analyzed. The predictive value was defined as the accuracy rate of preoperative prediction greater than or equal to $80 \%$.

Statistical analysis

Statistical analysis was performed using SPSS 25.0 (IBM, Chicago). The accuracy of prediction is expressed by rate. The distance is represented by the mean and standard deviation. 


\section{Results}

MSCT data of 80 patients were reconstructed and analyzed as required. Of the 80 patients,74(92.5\%)patients were able to locate the ICV and ICA roots and the anatomical relationship between them, of which 72 were confirmed by surgical findings,and the accuracy was $90 \%(72 / 80)$. The location of GTH relative to the pancreas was founded in 75(93.7\%)patients, and the accuracy was 91.25\%(73/80). ICV-GTH distance was measured in 73(91.2\%)patients, and the mean distance was 4.28 $\pm 2.5 \mathrm{~cm}$. The leSMA-reSMV distance was measured in 76(95\%)patients, and the mean distance was 2.21 $\pm 0.6 \mathrm{~cm}$.

Table 2

Results $(n=80)$

\begin{tabular}{|ll|}
\hline & $\mathrm{n}(\%)$ \\
\hline ICV \& ICA roots both located & $74(92.5 \%)$ \\
\hline ICA located in the front of ICV & $43(59.7 \%)$ \\
\hline ICA located in the rear of ICV & $29(40.3 \%)$ \\
\hline Prediction accuracy of the relationship between ICA and ICV & $72(90 \%)$ \\
\hline Location of GTH relative to the pancreas founded & $75(93.7 \%)$ \\
\hline Prediction accuracy of location of GTH & $73(91.25 \%)$ \\
\hline MCA roots located & $47(58.75 \%)$ \\
\hline Prediction accuracy of location of MCA & $37(46.25 \%)$ \\
\hline MCV roots located & $51(63.75 \%)$ \\
\hline Prediction accuracy of location of MCV & $43(53.75 \%)$ \\
\hline ICV-GTH distance (mean \pm SD) & $4.28 \pm 2.5 \mathrm{~cm}$ \\
\hline IeSMA-reSMV distance (mean \pm SD) & $2.21 \pm 0.6 \mathrm{~cm}$ \\
\hline
\end{tabular}

\section{Discussion}

Right hemicolectomy could be performed through different approaches: cephalic approach, caudal approach, and central approach. Among them, the central approach is usually considered the most consistent with the principle of radical resection of tumors, which requires first dissection and ligation of the root vessels in the superior mesenteric vascular region. However $\llbracket d u e$ to the vascular variations in the superior mesenteric region ${ }^{10-13}$, it is not easy for surgeons to expose root vessels and dissect lymph nodes in the central ligation region during surgery־especially in laparoscopic ones and patients with high BMI. 
Basically,in the right hemicolectomy with a central approach, the surgeons first need to look for ileocolic vessels and separates the roots of the ICV and ICA and ligates them separately.

In CME with CVL operations,ligation of ICV and right colic vein (if exists) was performed along the right border of the SMV. Then when dissected cephalad, GTH will be encountered in most patients. In the case of unclear anatomy, intraoperative bleeding mostly results from damage to these vessels, especially the ICV and GTH, which can lead to massive bleeding.

The left margin of SMA is the boundary of CME operations, and only when this margin is reached can sufficient lymph node dissection be achieved. However, in patients with high BMI, it is difficult to determine the boundary because the main blood vessels are usually covered by thickened mesenteric adipose tissue.

In conclusion,the key points of CME with CVL operations are to locate the root of ICV, ICA, GTH, the right edge of SMV, and the left edge of SMA. In order to reduce these difficulties in right hemicolectomy, this study attempts to evaluate the accuracy of MSCT with coronal reconstruction in predicting important anatomical sites preoperatively.

MSCT portal venous phase data were used for coronal reconstruction because these data can simultaneously show the main veins and arteries of the right hemicolectomy, including ICV, ICA, GTH, SMV, and SMA. Preoperative visualization of these vessels can be used to determine the extent of the $\mathrm{CVL}$ region during the right hemicolectomy.

In this study, of the 80 patients,74(92.5\%) patients were able to locate the ileocolic vein and artery roots and analyze the anatomical relationship between them, of which 72 were confirmed by surgical findingsICA crossed $43(59.7 \%)$ in front of ICV and $29(40.3 \%)$ in the rear of ICV-and the accuracy was $90 \%$ (72/80). These findings could guide the initial stage of the right hemicolectomy via a central approach. For example, in the case of which ICA crosses in the rear of ICV, ligation and lymph node dissection of ICA root need to be carried out behind SMV.

To determine the anatomical relationship between ICV and ICA, in addition to analyzing the coronalreconstructed image, it's needed to analyze the axial image combined with the MSCT positioning function simultaneously to increase the accuracy (Fig. 1).

GTH was located by MSCT in 75(93.7\%)patients, and the accuracy was $91.25 \%(73 / 80)$. These findings could help us predict the surgical route along SMV and may reduce the occurrence of intraoperative vascular injury and bleeding.

When we located the roots of ICV and GTH, we were able to measure the linear distance from the root (lower margin) of the ICV to the root (lower margin) of the GTH (Fig. 2). In this study, ICV-GTH distance was measured in $73(91.2 \%)$ patients, and the mean distance was $4.28 \pm 2.5 \mathrm{~cm}$. 
Meanwhile, by analyzing coronal-reconstructed images, we can determine the left edge of SMA (leSMA) and the right edge of SMV (reSMV), and then measure the maximum distance between them (Fig. 3). Coronal-reconstructed images revealed $93.7 \%(75 / 80)$ edges of SMA and SMV. The leSMA-reSMV distance was measured in $75(93.7 \%)$ patients, and the mean distance was $2.21 \pm 0.6 \mathrm{~cm}$. Combining these findings, we can estimate the size of the central ligation region.

Basically, in performing complete mesocolic excision with central vascular ligation, dissection along the left border of the SMA is required, but intuitive observation during operation could be difficult in patients with high BMI. As showed above,reconstructed coronal MSCT images could help to predict this border.

In summary, MSCT with coronal reconstruction technology makes it possible to predict the critical points of right hemicolectomy preoperatively. To reconstruct these images, patients only need conventional abdominal contrast-enhanced MSCT scan, without additional angiography. It makes this technology more suitable for clinical application, especially for those hospitals restricted in equipment or technology, and for patients who are afraid of increasing the radiation dose they receive.

Besides, coronal reconstruction can also help surgeons to detect vascular anatomical variations preoperatively, as shown in the following figure (Fig. 4): A patient's ICV flows directly into GTH and, if not noticed, may cause intraoperative injury or bleeding.

Compared with angiography, MSCT with coronal reconstruction technique also retains more perivascular anatomical information, such as the relative location of the pancreas, tumors, duodenum, which can guide the operation of right hemicolectomy.

However,this study also found that this technique may not be suitable for locating the middle colon vessels and right colic vessels as the detection rate of these vessels is low, mainly because these vessels are too small in diameter to be found by conventional enhanced MSCT scans.

\section{Conclusions}

MSCT with coronal reconstruction,can locate the roots of ICV, ICA, and GTH, predict the boundary of SMA and SMV, analyze the anatomical relationship between them, and estimate the location and range of the central ligation region. In conclusion, MSCT with coronary reconstruction can help surgeons obtain visualizable information and are of some predictive value in central vascular ligation in the right hemicolectomy with complete mesocolic excision.

\section{Abbreviations}

CME: complete mesocolic excision; CVL: central vascular ligation; MSCT: multi-slice spiral computed tomography; ICV: ileocolic vein; ICA: ileocolic artery; GTH: the gastrocolic trunk of Henle; ICV-GTH distance: distance from the ICV root to the GTH root; leSMA-reSMV distance: maximum distance from the left edge of the superior mesenteric artery to the right edge of the superior mesenteric vein; $\mathrm{PH}$ : pancreas 
head; MDCT: multidetector computed tomography angiography; 3D-CT: three-dimensional CT; BMI: body mass index.

\section{Declarations}

\section{Acknowledgments}

We thank the patients enrolled in this study.

\section{Authors' contributions}

1 Guarantor of integrity of the entire study: Hui Yu. 2 Study design: Hui Yu;Chunkang Yang. 3 Implementation research: Hui Yu; Yong Zhuang; Jinliang Jian. 4 Data and statistical analysis: Jinliang Jian; Yong Zhuang. 5 Manuscript writing: Hui Yu. The authors read and approved the final manuscript.

\section{Funding}

This submission was not funded.

\section{Availability of data and materials}

All data generated or analyzed during this study are included in this published article.

\section{Ethics approval and consent to participate}

All patients' signed informed consent was provided. Ethics approval was not required because patient's privacy information was not used in this retrospective study.

\section{Patient consent for publication}

Patients provided consent for the publication of the present study.

\section{Competing interests}

The authors declare that they have no competing interests.

\section{References}

1. W H, K W, K M, T P, S M. Standardized surgery for colonic cancer: complete mesocolic excision and central ligation-technical notes and outcome. Colorectal disease : the official journal of the Association of Coloproctology of Great Britain and Ireland. 2009;11: 354-364; discussion 364-355.

2. S T, H O, S A. Rationale for extent of lymph node dissection for right colon cancer. Diseases of the colon and rectum. 1995;38: 705-711. 
3. Y H, K M, Y S, et al. Japanese Society for Cancer of the Colon and Rectum (JSCCR) guidelines 2019 for the treatment of colorectal cancer. International journal of clinical oncology. 2020;25: 1-42.

4. NP W, H K, K T, et al. Understanding optimal colonic cancer surgery: comparison of Japanese D3 resection and European complete mesocolic excision with central vascular ligation. Journal of clinical oncology : official journal of the American Society of Clinical Oncology. 2012;30: 1763-1769.

5. Nesgaard JM, Stimec BV, Bakka AO, Edwin B, Ignjatovic D, group RCCs. Navigating the mesentery: a comparative pre- and per-operative visualization of the vascular anatomy. Colorectal Dis. 2015;17: 810-818.

6. M S, BV S, JF F, S T, D I. 3D relations between right colon arteries and the superior mesenteric vein: a preliminary study with multidetector computed tomography. Surgical endoscopy. 2011;25: 18831886.

7. S R, L R. Utilization patterns of multidetector computed tomography in elective and emergency conditions: indications, exposure risk, and diagnostic gain. Seminars in ultrasound, CT, and MR. 2010;31: 53-56.

8. I I, N K, M K. The computerized tomography scans and their dosimetric safety. Hellenic journal of nuclear medicine. 2008;11: 82-85.

9. CL S, KM H, EK F. Mesenteric CT angiography: a discussion of techniques and selected applications. Techniques in vascular and interventional radiology. 2006;9: 150-155.

10. C W, K Y, Y W, et al. Variations in right colic vascular anatomy observed during laparoscopic right colectomy. World journal of surgical oncology. 2019;17: 16.

11. D I, S S, B S, R B. Vascular relationships in right colectomy for cancer: clinical implications. Techniques in coloproctology. 2007;11: 247-250.

12. R P, G L, G P, M S, V S, L B. Gastrocolic trunk of Henle and its variants: review of the literature and clinical relevance in colectomy for right-sided colon cancer. Surgical and radiologic anatomy : SRA. 2019;41: 879-887.

13. SJ L, SC P, MJ K, DK S, JH O. Vascular Anatomy in Laparoscopic Colectomy for Right Colon Cancer. Diseases of the colon and rectum. 2016;59: 718-724.

\section{Figures}




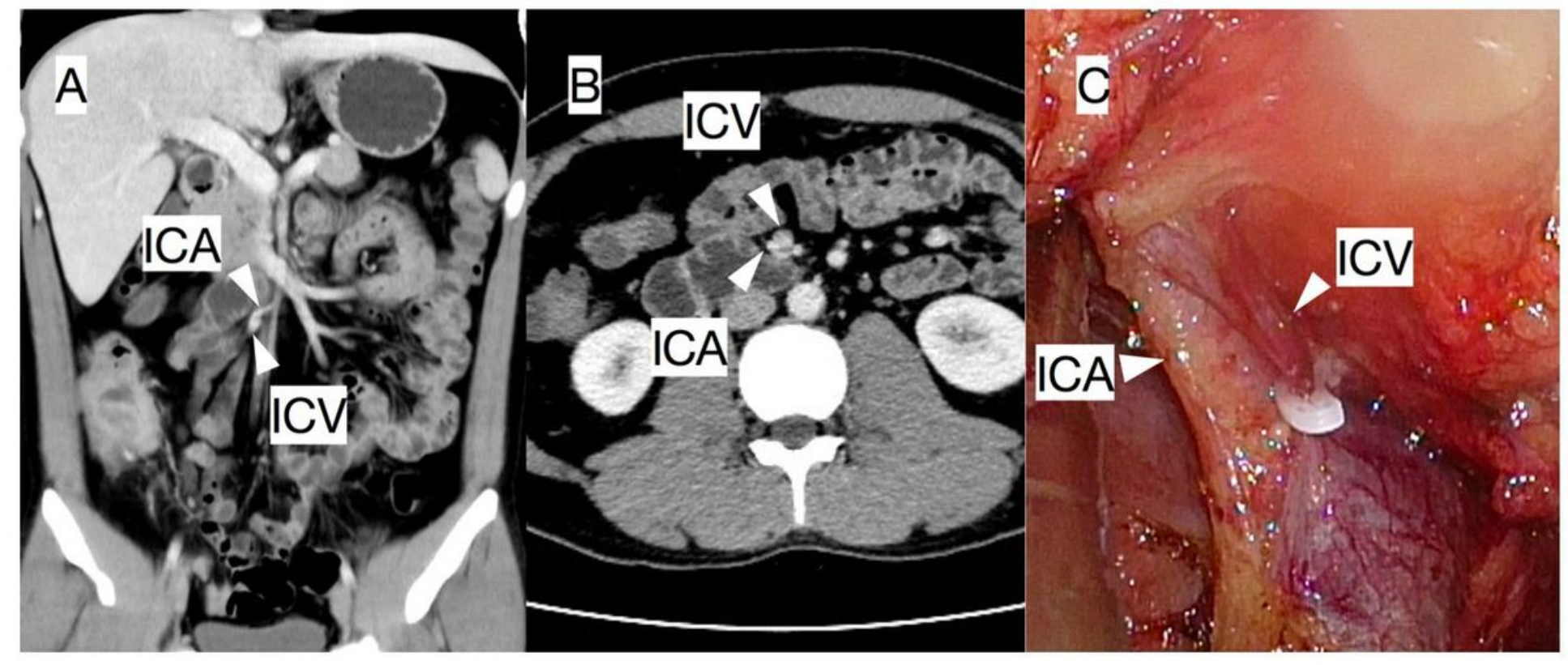

Figure 1

In a 34 years old male patient whose ICA was in the rear of ICV, the anatomical relationship between ICA and ICV was determined preoperatively by cross-analysis of coronal(A) and axial images(B) \which was proved by intraoperative findings $₫ C$ ).

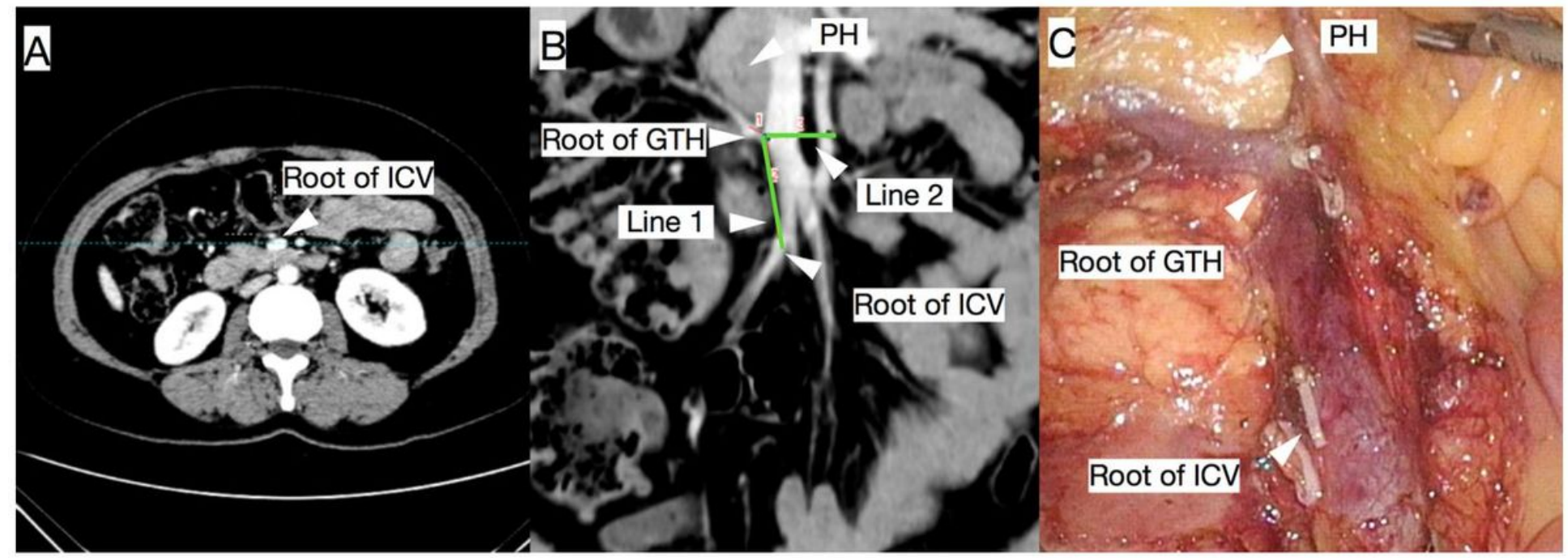

Figure 2

By analyzing the axial(A) and coronal(B) MSCT images of a 49 yeas old female patient, we were able to locate the root of GTH and ICV, find the location of the pancreas head $\triangle \mathrm{PH} \bigotimes$, and measure the ICV-GTH distance (Line1). The anatomical relationship between these structures could be used as guidance during surgery (C). 


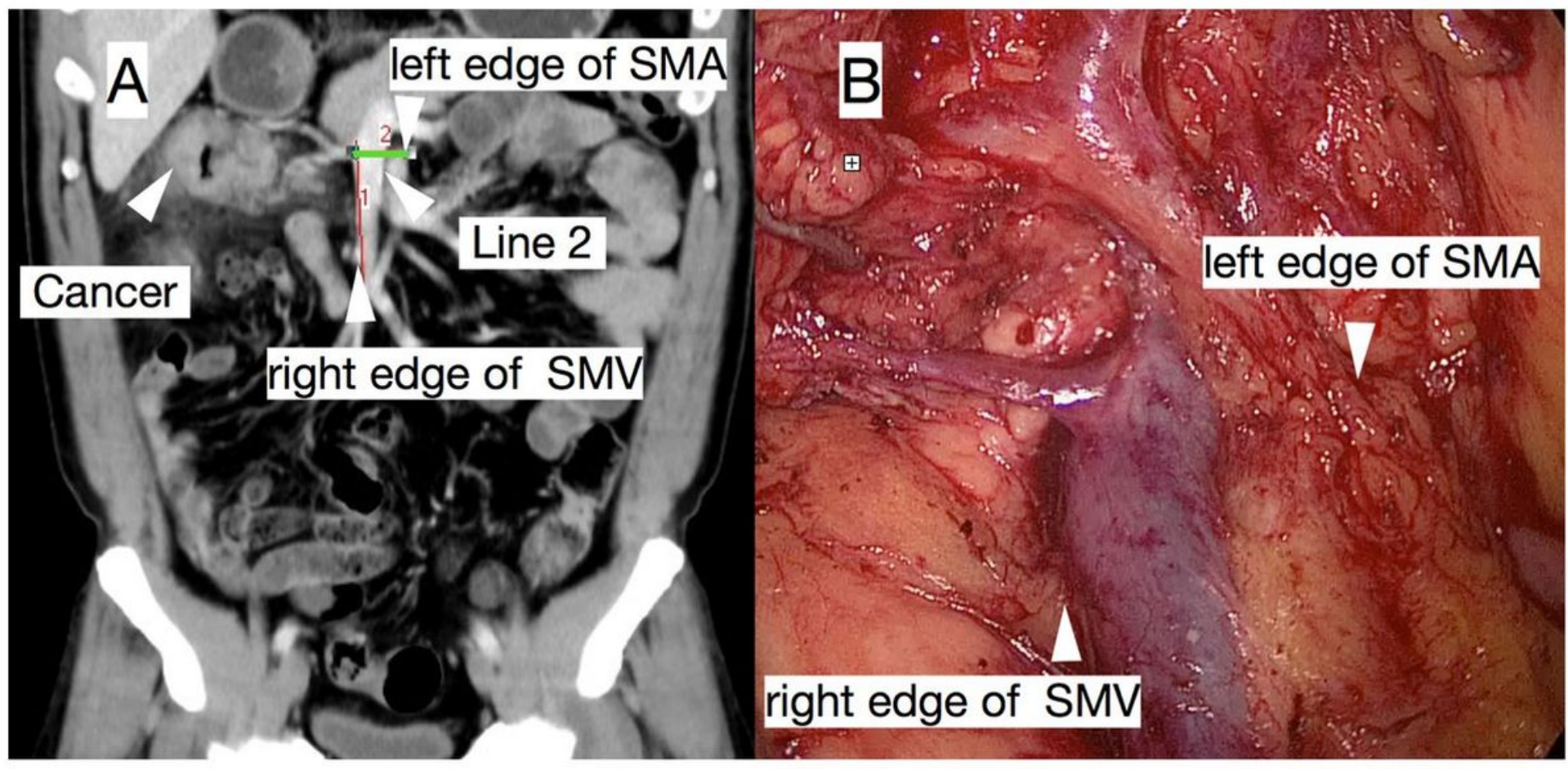

Figure 3

In a 42 years old patient with hepatic flexure colon cancer, the left edge of SMA and the right edge of SMV were located by coronal-reconstructed images (A), and the leSMA-reSMV distance(Line2) was measured, which was proved by intraoperative findings(B).

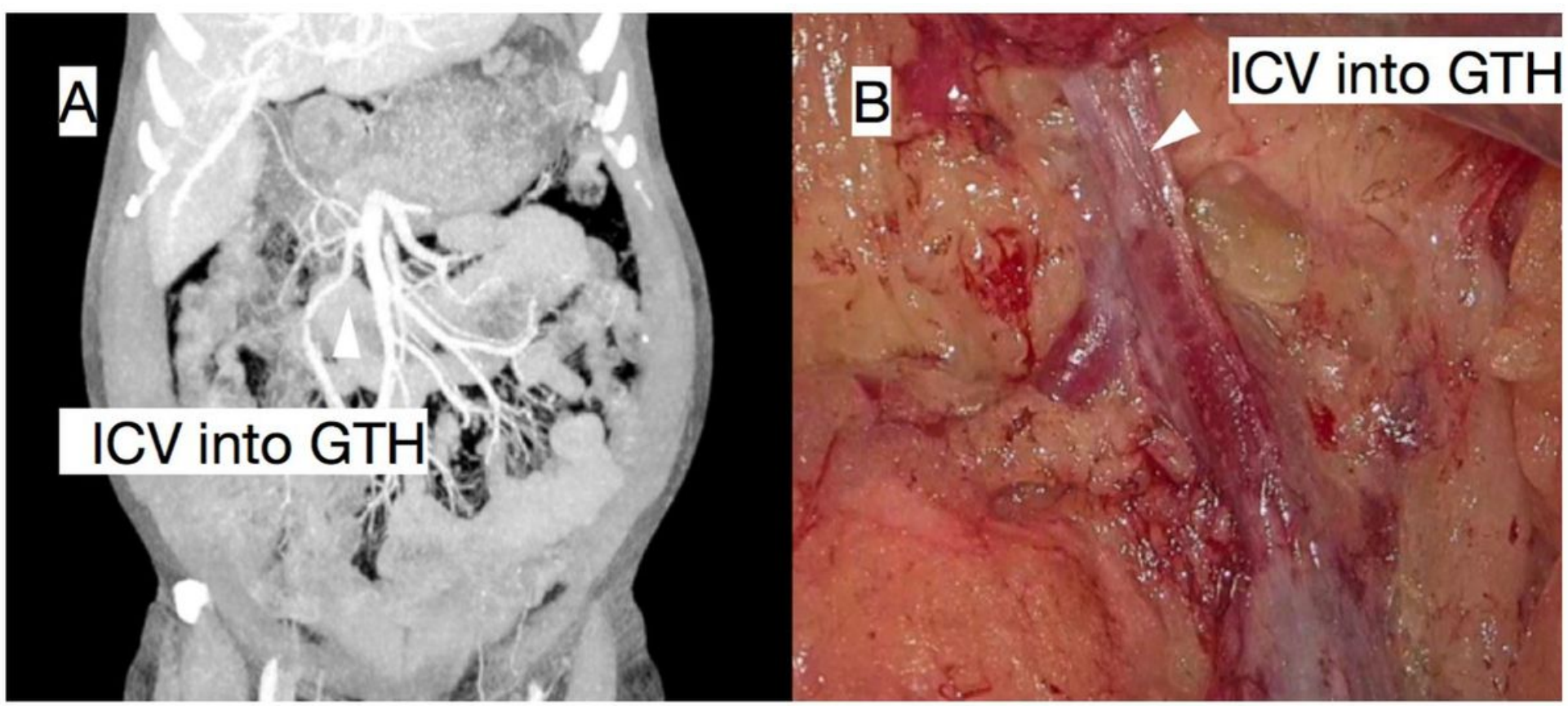

Figure 4

In a 62 years old male patient with proximal transverse colon cancer, MIP reconstruction on the coronal plane(A) and intraoperative images(B) showed that ICV directly flowed into GTH. 